\title{
Durable response to bevacizumab in adults with recurrent pilocytic astrocytoma
}

Andrea Wasilewski*,1 \& Nimish Mohile**,1

${ }^{1}$ Department of Neurology, University of Rochester, Box 704, 601 Elmwood Avenue, Rochester, NY 14642, USA

* Author for correspondence: andrea_wasilewski@urmc.rochester.edu

** Author for correspondence: Tel.: +01 585276 2463; Fax: +01 585276 3972; nimish_mohile@urmc.rochester.edu

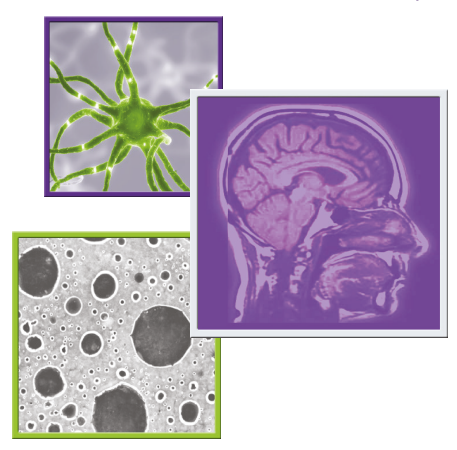

Practice points

- Pilocytic astrocytoma tend to be more aggressive in adults than children with higher recurrence rates.

- No standard of care currently exists for treatment of adult pilocytic astrocytoma (APA).

- APA are highly vascular tumors with high levels of VGEF expression that may be uniquely sensitive to antiangiogenic treatments such as bevacizumab (BEV).

- Antiangiogenic treatment with BEV produced durable clinical and radiographic response in four adults with recurrent APA.

- BEV may have true antitumor activity and survival benefit in APA, unlike in adult high-grade gliomas.

- Multicenter prospective studies are warranted to further assess efficacy of BEV in recurrent APA.

Background: Adultpilocytic astrocytomas are rare and highly vascular tumors. Aim: We hypothesized that they may be uniquely responsive to bevacizumab (BEV). Patients: We present four adult patients with pathologically diagnosed WHO grade I pilocytic astrocytoma who had robust and durable responses to BEV at time of recurrence. Three patients developed radiographic changes on MRI, consistent with progressive disease based on response assessment in neuro-oncology criteria. Median time to recurrence was 8.5 months. Methods: All patients were treated with six cycles of BEV for recurrence. Results: At the end of treatment, all patients had achieved a clinical and radiographic response. Median follow-up time after BEV is 20.5 months. Conclusion: This suggests that BEV may have true antitumor activity in adult pilocytic astrocytomas and may be important for achieving durable disease control.

First draft submitted: 29 December 2017; Accepted for publication: 2 March 2018; Published online: 9 April 2018

Keywords: astrocytoma $\bullet$ brain tumor $\bullet$ CNS neoplasms

Adult pilocytic astrocytomas (APA) are rare tumors with an incidence of 3.4 per 1 million [1]. These tumors usually arise from deep midline structures as well the brainstem and cerebellum. Juvenile piloytic astrocytomas occurring in children are curable with complete surgical resection [2]. Very few studies have prospectively investigated pilocytic astrocytomas in adults, but some retrospective studies and anecdotal reports suggest that APAs have a more aggressive clinical course when compared with Juvenile piloytic astrocytomas, with a higher incidence of tumor recurrence and progression [3]. Recurrence rates for APA have been reported as high as 30-42\% [4]. Currently, no standard of care exists for treatment of APA, especially following recurrence.

Bevacizumab (BEV) is a humanized monoclonal antibody directed against VEGF. The blocking of VEGF pathways has been demonstrated to decrease the permeability of vasculature and decrease perilesional edema in glioblastoma [5]. Several studies have shown no overall survival benefit with BEV in glioblastoma [6,7], although antiangiogenic treatment alone has not been studied in APA. One case series demonstrated clinical and radiographic improvement with the combination of BEV and temozolomide (TMZ) [8]. TMZ is an alkylating chemotherapeutic agent that penetrates the blood-brain barrier and is currently approved for treatment of malignant glioma [9]. APA are known to be highly vascular tumors with high levels of transcription and expression of VEGF and its receptors [10]. Given their vascularity, we hypothesized that APA may be uniquely responsive to treatments that block VEGF such as BEV. 
Table 1. Patient characteristics.

\begin{tabular}{|c|c|c|c|c|}
\hline Characteristics & Patient 1 & Patient 2 & Patient 3 & Patient 4 \\
\hline Age at diagnosis (years) & 66 & 43 & 25 & 23 \\
\hline Tumor location & Cerebellar vermis & Lateral ventricle & Tectum, thalamus & Brainstem \\
\hline Degree of surgical resection & Partial & Near total & Biopsy & Partial \\
\hline Pathology & $\begin{array}{l}\text { Atypical pilocytic astrocytoma, } \\
\text { WHO grade I }\end{array}$ & $\begin{array}{l}\text { Pilocytic astrocytoma, WHO } \\
\text { grade I }\end{array}$ & $\begin{array}{l}\text { Pilocytic astrocytoma, WHO } \\
\text { grade I }\end{array}$ & $\begin{array}{l}\text { Pilocytic astrocytoma, WHO } \\
\text { grade I }\end{array}$ \\
\hline \multicolumn{5}{|l|}{ Immunochemical tumor features } \\
\hline$B R A F$ mutation & Negative & Negative & Negative & Negative \\
\hline$I D H$ mutation & Negative & Negative & Negative & Negative \\
\hline Ki-67 proliferation index (\%) & 9 & 3 & 7 & 5 \\
\hline Initial treatment & $\begin{array}{l}\text { Radiation } \\
\text { Concurrent TMZ }\end{array}$ & $\begin{array}{l}\text { Radiation } \\
\text { Adjuvant TMZ }\end{array}$ & $\begin{array}{l}\text { Radiation } \\
\text { Steroids }\end{array}$ & $\begin{array}{l}\text { Radiation } \\
\text { Steroids }\end{array}$ \\
\hline $\begin{array}{l}\text { Time from diagnosis to } \\
\text { recurrence (months) }\end{array}$ & 4 & 7 & 10 & $11^{\dagger}$ \\
\hline
\end{tabular}

\section{Case series}

We present a series of four consecutive adult patients with pathologically diagnosed WHO grade I pilocytic astrocytoma who had a robust and durable response to BEV at time of recurrence. The median age at diagnosis was 34 years (range: 23-66). Three tumors were located infratentorially (cerebellum, tectum and brainstem) and one in the lateral ventricle. Two patients underwent a partial resection, one a near total resection and one patient underwent biopsy only. Two patients developed hydrocephalus requiring placement of ventricular shunts. BRAF $v 600 E$ mutation and $I D H$ mutation were absent in all patients. Ki-67 proliferation index was 5-9\%. Initial treatment was radiation alone in three patients and concurrent radiation and TMZ in one patient (Table 1).

\section{Patient 1}

A 66-year-old man presented with gait instability, vertigo, nausea and cognitive decline. Brain MRI showed a contrast enhancing cerebellar vermian lesion with mass effect on the fourth ventricle and obstructive hydrocephalus. He underwent a partial tumor resection with persistent diplopia postoperatively. Pathology was consistent with a pilocytic astrocytoma with atypical features and detection of KIAA1549-BRAF fusion. His MGMT promoter methylation status was indeterminate. He underwent treatment with concurrent radiation and TMZ. His course was complicated by a pseudomeningocele and required placement of a ventricular shunt. 4 months later, MRI brain with gadolinium showed a significant increase in APA contrast enhancement and a new region of nodularity. Given his worsening symptoms and performance status he was not a candidate for surgical re-resection.

\section{Patient 2}

A 43-year-old man presented with bilateral progressive hearing loss, tinnitus and headaches. Brain MRI showed a contrast-enhancing mass within the frontal horn of the right lateral ventricle with mild hydrocephalus. $\mathrm{He}$ underwent a near total resection of the mass without complications. Pathology was consistent with a pilocytic astrocytoma. He underwent radiation therapy alone. Repeat brain MRI with gadolinium seven months later showed significant interval increase of tumor enhancement, as well as new, irregular enhancing ependymal foci. He was then started on treatment with TMZ, which was complicated by thrombocytopenia.

\section{Patient 3}

A 25-year-old female presented with new onset headaches, nausea, emesis and left-sided numbness. Brain MRI demonstrated a contrast enhancing right dorsolateral tectal mass involving the right thalamus with moderate compression of the brainstem and acute obstructive hydrocephalus. A ventricular shunt was successfully placed to alleviate the hydrocephalus. She underwent biopsy of the tumor and pathology was consistent with pilocytic astrocytoma. Given the tumor location she was not a candidate for resection and was initially treated with 6 weeks of radiation therapy and steroids. 10 months later she developed diplopia, ptosis and worsening headaches, which 

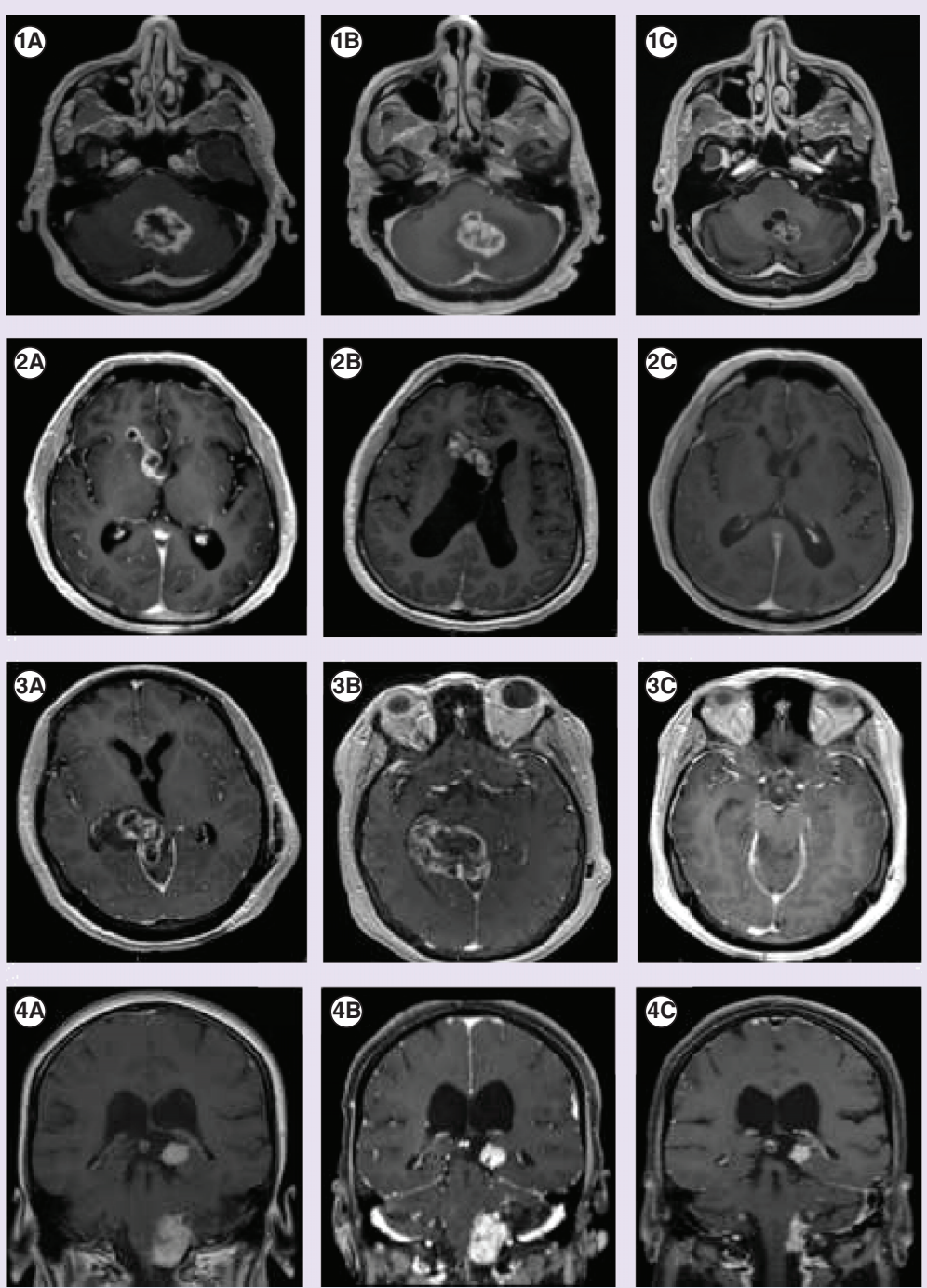

Figure 1. MRI brain at time of diagnosis, recurrence and post-treatment with bevacizumab.

Patient 1: (A) Axial T1 MRI with gadolinium at time of diagnosis showing APAs (Adult pilocytic astrocytomas) in the cerebellar vermis. (B) Axial T1 MRI with gadolinium at time of recurrence with increased contrast enhancement and tumor size. (C) Axial T1 MRI with gadolinium following treatment with six cycles of bevacizumab showing reduction of tumor size and enhancement.

Patient 2: (A) Axial T1 MRI with gadolinium at time of diagnosis showing APA in the right lateral ventricle. (B) Axial T1 MRI with gadolinium at time of recurrence with increased contrast enhancement and tumor extension. (C) Axial T1 MRI with gadolinium following treatment with six cycles of bevacizumab showing resolution of tumor enhancement. Patient 3: (A) Axial T1 MRI with gadolinium at time of diagnosis showing APA in the right thalamus and tectum. (B) Axial T1 MRI with gadolinium at time of recurrence with increased tumor contrast enhancement and new region of nodularity. (C) Axial T1 MRI with gadolinium following treatment with six cycles of bevacizumab showing resolution of enhancement and no evidence of tumor recurrence.

Patient 4: (A) Coronal T1 MRI with gadolinium at time of diagnosis showing APA in the pons and left thalamus. (B) Coronal TI MRI with gadolinium persistent tumor contrast enhancement. (C) Coronal T1 MRI with gadolinium

following treatment with six cycles of bevacizumab showing tumor regression and resolution of tumor enhancement.

were refractory to steroid therapy. MRI brain with gadolinium at that time showed increased contrast enhancement and tumor extension. 


\section{Patient 4}

A 23-year-old female presented with new onset headaches in the setting of pregnancy. MRI of the brain revealed a contrast enhancing anterior brainstem mass with surrounding vasogenic edema and an ependymal nodule lining the fourth ventricle. She underwent partial surgical resection without complications. Pathology was consistent with pilocytic astrocytoma. She underwent radiation therapy and was treated with multiple courses of steroids. Her course was complicated by multiple bi-hemisphreic infarcts as well as complications of prolonged steroid use including Cushing syndrome and thoracic compression fractures. She was unable to successfully taper off of steroids due to mass effect and recurrence of neurologic symptoms. Contrasted MRI brain eleven months following diagnosis showed persistent tumor enhancement and mass effect.

\section{Diagnosis/assessment}

Patients 1, 2 and 3 developed radiographic changes on MRI, including disease outside the radiation field, that were consistent with progressive disease based on Response Assessment in Neuro-Oncology (RANO) criteria (Figure 1) [11]. Patient 4 had persistent contrast enhancement of her tumor and increased perilesional edema on follow-up MRI brain, despite prior radiation. The median time to recurrence was 8.5 months (range: 4-10 months).

\section{Treatment/management}

All patients were treated with BEV $10 \mathrm{mg} / \mathrm{kg}$ every 14 days for six cycles at the time of recurrence. Treatment with BEV was well tolerated and no patients experienced adverse effects. One patient also received adjuvant TMZ. Following treatment with BEV, steroids were able to be weaned and discontinued in all patients.

\section{Outcome/implications}

At the end of treatment with BEV, all patients had achieved a significant clinical and radiographic response. MRI brain obtained after six cycles of BEV showed significant decrease or resolution of tumor contrast enhancement in all patients. All patients experienced a significant reduction in tumor size following BEV on follow-up brain MRI. Patients 1 and 3 experienced complete responses, with no radiographic evidence of tumor visualized on follow-up MRI. The median follow-up time after treatment with BEV is 22 months (range: 7-37). As of their last follow-up visit, no patient has recurred.

\section{Discussion/conclusion}

Although, BEV has failed to demonstrate an overall survival benefit to date in adult gliomas, it has been shown to prolong progression-free survival in glioblastoma [12]. This series suggests that BEV may have true antitumor activity in APA, improving both progression free and overall survival. Recurrence in APA in this series is consistent with previous reports. Sustained decrease in tumor size and contrast enhancement following BEV, suggest that it may be an important agent to achieve durable disease control. The imaging findings may have also had a component of radiation necrosis, which can spontaneously resolve. We believe that BEV at a minimum helped to hasten this response in addition to treatment of tumors outside of the radiation field. The unique features of APA including hyaline vessels and vascular proliferation may be affected by antiangiogenic treatment, resulting in decreased vascular permeability and proliferation and overall tumor reduction [13].

To date, there are no prospective studies evaluating the use of antiangiogenic treatments in pilocytic astrocytoma. This case series suggests that targeting angiogenesis may be a novel therapeutic approach in APA. Given the low incidence of this tumor, future multicenter studies to further assess efficacy are warranted.

\footnotetext{
Financial \& competing interests disclosure

The authors have no relevant affiliations or financial involvement with any organization or entity with a financial interest in or financial conflict with the subject matter or materials discussed in the manuscript. This includes employment, consultancies, honoraria, stock ownership or options, expert testimony, grants or patents received or pending, or royalties.

No writing assistance was utilized in the production of this manuscript.
}

Informed consent disclosure

The authors state that they have obtained verbal and written informed consent from the patient/patients for the inclusion of their medical and treatment history within this case report. 


\section{Open access}

This work is licensed under the Creative Commons Attribution 4.0 License. To view a copy of this license, visit http://creativecommons.org/licenses/by/4.0/

\section{References}

1. Ostrom Q, Gittleman H, Liao P et al. Central brain tumor registry U.S. statistical report: primary brain and central nervous system tumors diagnosed in the United States in 2007-2011. Neuro. Oncol. 16(Suppl. 4), iv1-iv63 (2014).

2. Burkhard C, Di Patre PL, Schuler D et al. A population-based study of the incidence and survival rates in patients with pilocytic astrocytoma. J. Neurosurg. 98(6), 1170-1174 (2003).

3. Stüer C, Vilz B, Majores M, Becker A, Schramm J, Simon M. Frequent recurrence and progression in pilocytic astrocytoma in adults. Cancer 110, 2799-2808 (2007).

4. Ellis JA, Waziri A, Balmaceda C, Canoll P, Bruce JN, Sisti MB. Rapid recurrence and malignant transformation of pilocytic astrocytoma in adult patients. J. Neurooncol. 95, 377-382 (2009).

5. Takano S, Kimu H, Tsuda K et al. Decrease in the apparent diffusion coefficient in peritumoral edema for the assessment of recurrent glioblastoma treated by bevacizumab. Acta Neurochir. Suppl. 118, 185-189 (2013).

6. Chinot OL, Wick W, Mason W et al. Bevacizumab plus radiotherapy - temozolomide for newly diagnosed glioblastoma. N. Engl. J. Med. 370, 709-722 (2014).

7. Gilbert MR, Dignam JJ, Armstrong TS. A randomized controlled trial of bevacizumab for newly diagnosed glioblastoma. N. Engl. J. Med. 370(8), 699-708 (2014).

8. Green R, Woyshner E, Quan J, Pope W, Cloughesy T. Treatment of unresectable adult pilocytic astrocytoma with bevacizumab with or without temozolomide. Neurology 78 (1 Suppl.), P04.18 (2012).

9. Freidman HS, Kerby T, Calvert H. Temozolomide and treatment of malignant glioma. Clin. Cancer Res. 6(7), 2585-2597 (2000).

10. Sie M, de Bont ES, Scherpen FJ, Hoving EW, den Dunnen WF. Tumor vasculature and angiogenic profile of paediatric pilocytic astrocytoma; is it much different from glioblastoma? Neuropathol. Appl. Neurobiol. 36(7), 636-647 (2010).

11. Van den Bent MJ, Wefel JS, Schiff D et al. Response assessment in neuro-oncology (a report of the RANO group): assessment of outcome in trails of diffuse low-grade glomas. Lancet Oncol. 12(6), 583-593 (2011).

12. Gilbert MR, Dignam JJ, Armstrong TS et al. A randomized trial of bevacizumab for newly diagnosed glioblastoma. N. Engl. J. Med. 370 , 699-708 (2014).

13. Leung SY, Chan AS, Wong MP, Cheung N, Chung LP. Expression of vascular endothelial growth factor and its receptors in pilocytic astrocytoma. Am. J. Surg. Pathol. 21(8), 941-950 (1977). 
